\title{
Urbanização da leishmaniose visceral: aspectos clínicos e epidemiológicos em Aracaju, Sergipe, Brasil
}

\author{
Urbanization of visceral leishmaniasis: clinical and epidemiological aspects in Aracaju, Sergipe state, Brazil \\ Urbanización de la leishmaniasis visceral: aspectos clínicos y epidemiológicos en Aracaju, Sergipe, Brasil
}

Marco Aurélio Oliveira Góes. Universidade Federal de Sergipe (UFS). Lagarto, SE, Brasil. marco.goes@saude.se.gov.br (Autor correspondente) Verònica de Lourdes Sierpe Jeraldo. Universidade Tiradentes (UNIT). Aracaju, SE, Brasil. veronica_sierpe@hotmail.com Alex Santana Oliveira. Faculdade Estácio de Sá (FASE). Aracaju, SE, Brasil. farmalexsantana@gmail.com

\section{Resumo}

Objetivo: Este estudo tem como objetivo descrever aspectos clínicos e epidemiológicos da Leishmaniose Visceral (LV) na cidade de Aracaju-SE, no período de 2007 a 2011. Métodos: Estudo epidemiológico retrospectivo, descritivo, utilizando dados oriundos do Sistema de Informação de Agravos de Notificação da Secretaria de Saúde do Estado de Sergipe, com análise estatística pelo Epi Info 6.04d. Resultados: Foram registrados 128 casos de LV no município, representando média anual de 25,6 casos, e um coeficiente de incidência de 23,5 casos para cada 100 mil habitantes. Houve predomínio do sexo masculino $(65,6 \%)$ e uma maior concentração de casos ocorreu em crianças até quatro anos $(26,6 \%)$. As manifestações clínicas mais encontradas foram: febre (96,1\%); esplenomegalia (89,1\%); hepatomegalia (79,7\%); fraqueza (82,0\%), e emagrecimento (77,3\%). 0 critério de confirmação foi clínico-laboratorial em 95,5\% dos casos. 0 coeficiente de letalidade geral no período estudado foi de 7,8\% (dez óbitos) e de 26,7\% em pessoas entre 40 e 49 anos (quatro óbitos). No período, foi identificado pelo menos um caso de LV em 65,8\% dos 38 bairros de Aracaju, distribuídos em todas as regiões da cidade. Conclusões: Verifica-se no estudo uma ampla distribuição da LV no município, além de uma alta letalidade. Para 0 enfrentamento adequado da LV, além das medidas relacionadas às intervenções ambientais e ao controle vetorial, é necessária a constante atualização dos profissionais de saúde, principalmente dos médicos da atenção primária e das unidades de pronto-atendimento, para que consigam identificar precocemente os casos suspeitos a fim de que o diagnóstico e o tratamento ocorram de forma oportuna.

\section{Abstract}

Objective: This study aims to describe the clinical and epidemiological aspects of visceral leishmaniasis (VL) in the city of Aracaju, Sergipe state, from 2007 to 2011. Methods: It is a descriptive retrospective epidemiological study using data from the Information System for Notifiable Diseases of the Health Department of Sergipe, with statistical analysis processed by the Epi Info 6.04d software. Results: One hundred twenty-eight cases of VL have been reported in the city of Aracaju, representing an annual average of 25.6 cases and an incidence rate of 23.5 cases per 100.000 inhabitants. There was predominance of males (65.6\%) and a higher number of cases occurred in children up to 4 years of age (26.6\%). The most frequent clinical manifestations were fever $(96.1 \%)$; splenomegaly (89.1\%); hepatomegaly $(79.7 \%)$; weakness $(82.0 \%)$, and weight loss $(77.3 \%)$. $95.5 \%$ of the cases were confirmed by clinical and laboratory criteria. The overall mortality rate during the study period was of $7.8 \%$ (10 deaths), and of $26.7 \%$ ( 4 deaths) in patients between 40 and 49 years of age. During the time of the study, at least one case of VL was identified in $65.8 \%$ of the 38 districts spread across all regions of the city. Conclusions: It was possible to verify a wide distribution of VL in the city, with high lethality. To deal with VL appropriately, besides adopting measures related to environmental interventions and vector control, it is necessary to constantly update health professionals, especially physicians working in primary care and emergency units, so that they can perform the early diagnosis of suspected cases and treatment can occur in a timely manner.

\section{Palavras-chave:} Leishmaniose Visceral Urbanização Vigilância Epidemiológica Letalidade

\section{Keywords:}

Leishmaniasis, Visceral Urbanization Epidemiological Surveillance Lethality

\section{Fonte de financiamento: declaram não haver. \\ Parecer CEP: Universidade Federal de Sergipe, parecer n. ${ }^{\circ} 143.953$. \\ Conflito de interesses: declaram não haver.


Resumen

Objetivo: este estudio tiene como objetivo describir los aspectos clínicos y epidemiológicos de la Leishmaniasis Visceral (LV) en la ciudad de Aracaju/ SE en el periodo comprendido de 2007 a 2011. Métodos: Se trata de un estudio epidemiológico retrospectivo descriptivo con datos del Sistema de Información de Enfermedades de Notificación del Departamento de Salud del Estado de Sergipe, y análisis estadístico realizado con el Epi Info 6.04d. Resultados: Se registraron 128 casos de LV en la ciudad de Aracaju, lo que representa una media anual de 25,6 casos y una tasa de incidencia de 23,5 casos por cada 100.000 habitantes. Hubo un predominio del sexo masculino $(65,6 \%)$ y una mayor concentración de casos se presentó en niños de hasta cuatro años (26,6\%). Las manifestaciones clínicas observadas fueron: fiebre (96,1\%); esplenomegalia (89,1\%); hepatomegalia $(79,7 \%)$; debilidad $(82,0 \%)$ y adelgazamiento $(77,3 \%)$. El criterio clínico fue confirmado en laboratorio en el $95,5 \%$ de los casos. La tasa de mortalidad durante el período de estudio fue de 7,8\% (10 muertes), y de 26,7\% en las personas entre 40 y 49 años (4 muertes). En dicho periodo se identificó por lo menos un caso de LV en el $65,8 \%$ de los 38 barrios de Aracaju, distribuidos por todas las regiones de la ciudad. Conclusiones: se verifica en el estudio una amplia distribución de la LV en la ciudad, además de una alta mortalidad. Para enfrentarse adecuadamente a la LV, más allá de las medidas relacionadas con las intervenciones ambientales y el control de vectores, es necesario una constante actualización de los profesionales de la salud, especialmente médicos tanto de atención primaria como de emergencia, para que puedan identificar precozmente los casos sospechosos con la finalidad de que el diagnóstico y el tratamiento ocurran de forma adecuada.
Palabras clave: Leishmaniasis Visceral Urbanización Vigilancia Epidemiológica Letalidad

\section{Introdução}

As leishmanioses constituem um grave problema de saúde pública em várias partes do mundo. Atualmente, estas ocorrem em quatro continentes e são consideradas endêmicas em 88 países, dos quais 72 são países em desenvolvimento. ${ }^{1}$ $\mathrm{Na} 60^{a}$ Assembleia Mundial de Saúde, as leishmanioses foram colocadas entre as doenças tropicais mais negligenciadas, considerando-se que 350 milhóes de pessoas estão em área de risco. ${ }^{1}$

A Leishmaniose Visceral (LV) ou Calazar é uma doença fatal, se não tratada, e está presente em 70 países, com os maiores focos na Ásia Meridional, com cerca de 300 mil casos/ano, seguida pela África Ocidental, com aproximadamente 30 mil casos/ ano. ${ }^{1,2}$ Cerca de $90 \%$ dos casos de leishmaniose visceral ocorrem em cinco países: Bangladesh, Brasil, Índia, Nepal e Sudão. ${ }^{1}$

Aproximadamente $90 \%$ dos casos registrados nas Américas ocorrem no Brasil, ${ }^{1,2}$ onde a LV está presente em 21 Estados da federação, atingindo todas as cinco regióes. ${ }^{3} \mathrm{Na}$ década de 1990 , cerca de $90 \%$ dos casos de LV ocorriam na Regiáo Nordeste, mas recentemente esta é responsável por $48 \%$ dos casos do país. ${ }^{3}$ Apesar de a Região Nordeste permanecer responsável pela maior concentração de casos, a LV tem se expandido e está presente em todas as regióes do país, causando surtos e apresentando, em alguns estudos, uma tendência ao aumento da letalidade. ${ }^{3,4}$

A urbanização da LV vem sendo bem documentada na literatura. ${ }^{5-8}$ Nas últimas três décadas, a sua transmissão tem sido descrita em várias cidades brasileiras de médio e grande porte. Acredita-se que este fenômeno resulte da ação antropogênica, com as rápidas alteraçóes ambientais, as migrações populacionais para as áreas urbanas periféricas e o aumento na interação com animais silvestres e cachorros, além da facilidade de adaptação do Lutzomya longipalpis ao ambiente peridomiciliar. ${ }^{8,9}$

Diferentemente de outras capitais, como Campo Grande, onde os primeiros casos são de $2001,{ }^{10} \mathrm{em}$ Aracaju, a primeira ocorrência foi em 1936, quando Evandro Chagas diagnosticou nessa cidade o primeiro caso da doença no Brasil. ${ }^{11}$ Observe-se que as características de distribuiçáo têm mudado, saindo da predominância da área de expansão (zona periurbana) para uma distribuição mais ampla, abrangendo áreas periféricas e centrais. Estudo recente demonstra abundância de Lutzomya longipalpis na capital sergipana, principalmente nos domicílios com presença de animais domésticos (cachorros e galinhas). ${ }^{12}$

Tendo em vista a expansão da LV, o objetivo deste estudo é descrever aspectos clínicos e epidemiológicos atuais desta doença em Aracaju, capital do Estado de Sergipe.

\section{Métodos}

Trata-se de um estudo epidemiológico retrospectivo descritivo com dados do Sistema de Informação de Agravos de Notificação (SINAN) da Diretoria de Vigilância Epidemiológica da Secretaria de Estado da Saúde de Sergipe, dos casos de LV residentes no município de Aracaju, capital de Sergipe, notificados de 1. ${ }^{\circ}$ de janeiro de 2007 a 31 de dezembro de 2011.

A população do estudo foi composta por todos os novos casos de LV, residentes no município de Aracaju, notificados entre 2007 e 2011. Foram excluídos os registros duplicados e as recidivas. Foram analisadas as variáveis constantes na ficha 
de notificação de LV: sexo, faixa etária, sinais e sintomas, critério de confirmação, coinfecção pelo HIV, exames laboratoriais específicos realizados e evolução clínica. Os dados foram extraídos do banco de dados SINAN da Diretoria de Vigilância Epidemiológica da Secretaria de Estado da Saúde de Sergipe, com a utilização do aplicativo TABWIN (DATASUS).

Para a análise dos dados, foram calculadas frequências, média, taxa de incidência e letalidade. Para calcular diferenças nas frequências, foi utilizado o teste do qui-quadrado de Pearson, considerando o nível de significância $\mathrm{p}<0,05$. Para a análise estatística, foi utilizado o software EPI INFO 6.04.d.

A pesquisa observou as recomendaçôes da Resoluçáo 196 de 10/10/1996 do Conselho Nacional de Saúde, sendo aprovada pelo Comitê de Ética da Universidade Federal de Sergipe com o parecer nº 143.953.

\section{Resultados}

Entre 2007 e 2011, foram notificados 128 novos casos de Leishmaniose Visceral (LV) no município de Aracaju, representando uma média anual de 25,6 casos. Entre os casos, houve predomínio do sexo masculino (65,6\%). A idade variou de sete meses a 69 anos, com média de 21,7 e mediana de 16,5 anos (desvio padrão de 18,7). A maior concentração de casos ocorreu em crianças de até quatro anos (26,6\%), havendo distribuição semelhante nas demais faixas etárias (Tabela 1).

O coeficiente de incidência médio da LV em Aracaju no período foi de 23,5 casos para cada 100 mil habitantes, sendo de 33,2 no sexo masculino e 15,1 no sexo feminino. A incidência entre os sexos variou conforme a faixa etária, sendo maior no sexo feminino nas faixas de ' 0 a 4 anos' $(89,2)$ e ' 5 a 9 anos' $(30,0)$, em relaçáo ao masculino (Tabela 1 ).

Tabela 1. Distribuição dos casos de Leishmaniose Visceral e coeficiente de incidência por faixa etária e sexo, Aracaju, Sergipe, 2007 a 2011.

\begin{tabular}{|c|c|c|c|c|c|c|c|c|c|}
\hline \multirow[t]{2}{*}{ Faixa Etária } & \multicolumn{3}{|c|}{ Masculino } & \multicolumn{3}{|c|}{ Feminino } & \multicolumn{3}{|c|}{ Total } \\
\hline & $\mathrm{n}$ & $\%$ & $\mathrm{Cl}$ & $\mathrm{n}$ & $\%$ & $\mathrm{Cl}$ & $\mathrm{n}$ & $\%$ & $\mathrm{Cl}$ \\
\hline 0 a 4 & 15 & 17,9 & 68,8 & 19 & 43,2 & 89,2 & 34 & 26,6 & 78,9 \\
\hline 5 a 9 & 6 & 7,1 & 25,8 & 7 & 15,9 & 30,0 & 13 & 10,2 & 27,9 \\
\hline 10 a 14 & 7 & 8,3 & 31,1 & 4 & 9,1 & 17,5 & 11 & 8,6 & 24,2 \\
\hline 15 a 19 & 12 & 14,3 & 51,8 & 2 & 4,5 & 8,4 & 14 & 10,9 & 29,7 \\
\hline 20 a 29 & 9 & 10,7 & 17,4 & 4 & 9,1 & 7,0 & 13 & 10,2 & 12,0 \\
\hline 30 a 39 & 11 & 13,1 & 27,0 & 4 & 9,1 & 8,2 & 15 & 11,7 & 16,8 \\
\hline 40 a 49 & 12 & 14,3 & 39,1 & 3 & 6,8 & 7,8 & 15 & 11,7 & 21,7 \\
\hline 50 e mais & 12 & 14,3 & 30,8 & 1 & 2,3 & 1,8 & 13 & 10,2 & 13,8 \\
\hline Total & 84 & 100,0 & 33,2 & 44 & 100,0 & 15,1 & 128 & 100,0 & 23,5 \\
\hline
\end{tabular}

$\mathrm{n}=$ número absoluto $/ \mathrm{Cl}=$ coeficiente de incidência (por 100 mil habitantes).

As manifestaçôes clínicas mais frequentes foram: febre (96,1\%); esplenomegalia (89,1\%); hepatomegalia (79,7\%); fraqueza $(82,0 \%)$ e emagrecimento $(77,3 \%)$. Além disso, alguns pacientes apresentavam quadro infeccioso associado (20,3\%), icterícia $(18,1 \%)$ e hemorragias $(15,6 \%)$ (Tabela 2$)$.

O critério de confirmação foi clínico-laboratorial em 95,5\% dos casos, sendo a reação de imunofluorescência indireta (RIFI) reagente em 90,2\% (92/102), o rk39 em 88,2\% (45/51) e a microscopia direta de punção de medula óssea em 66,0\% (31/47) (Tabela 3). O diagnóstico de 4,5\% dos casos foi baseado em critérios clínicos e epidemiológicos.

Dentre os casos notificados no período, houve registro da coinfecção pelo HIV em 4,6\% (seis casos), mas em 29,7\% (38) dos casos, a situação sorológica para HIV era desconhecida. A droga inicialmente utilizada em 78,1\% foi o antimoniato pentavalente $n$-metil glucamina (Glucantime ${ }^{\circledR}$ ), seguida pela anfotericina B desoxicolato $(17,2 \%)$ e anfotericina B lipossomal (4,8\%). Nos 12 pacientes com mais de 50 anos, foi utilizado o Glucantime ${ }^{\circledR}$ em 50\%, assim como em 70,0\% dos pacientes com icterícia, em que também foi utilizada essa droga. 
Tabela 2. Distribuição das variáveis clínico-epidemiológicas e letalidade dos casos de Leishmaniose Visceral em Aracaju, Sergipe, 2007 a 2011.

\begin{tabular}{|c|c|c|c|c|}
\hline \multirow[t]{2}{*}{ Variáveis } & \multicolumn{2}{|c|}{ Casos } & \multirow{2}{*}{$\begin{array}{c}\text { Óbitos } \\
n\end{array}$} & \multirow{2}{*}{$\begin{array}{c}\text { Letalidade } \\
\% \\
\end{array}$} \\
\hline & $n$ & $\%$ & & \\
\hline \multicolumn{5}{|l|}{ Sexo } \\
\hline M & 84 & 65,6 & 5 & 6,0 \\
\hline $\mathrm{F}$ & 44 & 34,4 & 5 & $11,4(p=0,22)$ \\
\hline \multicolumn{5}{|l|}{ Faixa Etária } \\
\hline 0 a 4 & 34 & 26,6 & 1 & 2,9 \\
\hline 5 a 9 & 13 & 10,2 & 0 & 0,0 \\
\hline 10 a 14 & 11 & 8,6 & 0 & 0,0 \\
\hline 15 a 19 & 14 & 10,9 & 1 & 7,1 \\
\hline 20 a 29 & 13 & 10,2 & 1 & 7,7 \\
\hline 30 a 39 & 15 & 11,7 & 1 & 6,7 \\
\hline 40 a 49 & 15 & 11,7 & 4 & $26,7(p=0,012)$ \\
\hline 50 e mais & 13 & 10,2 & 2 & 15,4 \\
\hline \multicolumn{5}{|l|}{ Sinais e Sintomas } \\
\hline Febre & 123 & 96,1 & 9 & 7,3 \\
\hline Fraqueza & 105 & 82,0 & 9 & 8,6 \\
\hline Edema & 31 & 24,2 & 6 & $19,4(p=0,013)$ \\
\hline Emagrecimento & 99 & 77,3 & 9 & 9,1 \\
\hline Palidez & 95 & 74,2 & 8 & 8,4 \\
\hline Esplenomegalia & 114 & 89,1 & 10 & 8,8 \\
\hline Quadro infeccioso & 26 & 20,3 & 4 & $15,4(p=0,07)$ \\
\hline Hemorragias & 20 & 15,6 & 4 & $20,0(p=0,017)$ \\
\hline Hepatomegalia & 102 & 79,7 & 7 & 6,9 \\
\hline Icterícia & 23 & 18,0 & 6 & $26,1(p=0,001)$ \\
\hline Tosse/diarreia & 80 & 62,5 & 6 & 7,5 \\
\hline Casos de LV & 128 & 100 & 10 & 7,8 \\
\hline
\end{tabular}

A taxa de letalidade no período estudado foi de 7,8\% (dez óbitos), sendo maior em pessoas entre 40 e 49 anos (26,7\%).

A letalidade foi maior no sexo feminino $(11,4 \%)$ do que no sexo masculino $(6,0 \%)$. A letalidade foi significativamente

Tabela 3. Distribuição do resultado das técnicas laboratoriais utilizadas para confirmação dos casos de Leishmaniose Visceral em Aracaju, Sergipe, 2007 a 2011.

\begin{tabular}{|c|c|c|c|c|c|c|}
\hline \multirow[t]{2}{*}{ Resultado } & \multicolumn{2}{|c|}{ RIFI } & \multicolumn{2}{|c|}{ rK39 } & \multicolumn{2}{|c|}{ Parasitológico } \\
\hline & $\mathrm{n}$ & $\%$ & $\mathrm{n}$ & $\%$ & $\mathrm{n}$ & $\%$ \\
\hline Positivo & 92 & 90,2 & 45 & 88,2 & 31 & 66,0 \\
\hline Negativo & 10 & 9,8 & 6 & 11,8 & 16 & 34,0 \\
\hline Total realizado & 102 & 100,0 & 51 & 100,0 & 47 & 100,0 \\
\hline
\end{tabular}

$\mathrm{RIFI}=$ Reação de Imunofluorescência Indireta; rk39 = teste qualitativo para detecção de anticorpos antileishmania que utiliza a proteína recombinante K39.

mais elevada nos pacientes com icterícia $(26,1 \%)$ e na presença de hemorragias $(20,1 \%)$ e edema $(19,4 \%)$, em relação aos demais $(p<0,05)$ (Tabela 2).

Quanto ao local de diagnóstico, 51,6\% foram realizados no Hospital de Urgência de Sergipe; 32,0\% no Hospital Universitário; 9,7\% nas Unidades de Saúde da Família; 4,7\% em hospitais particulares, e 2,5\% em unidades de prontoatendimento municipais.

Nos cinco anos estudados, 65,8\% (25) dos 38 bairros de Aracaju apresentaram pelo menos um caso de LV, distribuídos por todas as regióes da cidade, sendo a maior concentração de casos nos bairros Santos Dumont (15,6\%), Mosqueiro $(10,9 \%)$ e América $(8,6 \%)$. 


\section{Discussão}

O município apresentou no período estudado uma média de 25,6 casos por ano (variando de 17 a 39 casos/ano), maior do que no estudo realizado anteriormente (1999-2008), que demonstrava uma média anual de 19,2 casos/ano, mas mantendo a classificação como área de transmissão intensa, pelos parâmetros do Ministério da Saúde.,13

Assim como em outros estudos, a LV tem sido mais frequente em crianças de até quatro anos, mas observa-se uma distribuição homogênea nas demais faixas etárias (Tabela 1). Alguns autores têm identificado um pico também entre adultos jovens. ${ }^{5,14,15}$

Foi identificada uma maior proporção no sexo masculino, principalmente nas faixas etárias acima de dez anos. ${ }^{5,7}$ No Brasil, a doença é mais frequente em menores de dez anos (58\%) e o sexo masculino é, proporcionalmente, o mais afetado (61\%). ${ }^{3}$ As razóes para afetar principalmente as crianças têm sido atribuídas a sua relativa imaturidade imunológica, associada à desnutriçãoo (comuns em áreas endêmicas), além da grande exposição dessa população ao ambiente peridomiciliar. ${ }^{3,15}$

Estudo com pacientes internados por LV na Espanha demonstrou que a maioria dos casos também era do sexo masculino (73\%), mas com média de idade de 33,5 anos. Essa maior média etária pode ser atribuída ao alto coeficiente de coinfecção pelo HIV (37\%) naquela casuística, visto que, nos HIV negativos, os autores encontraram uma maior concentração em menores de dez anos, principalmente menores de cinco anos. ${ }^{16}$

A LV é uma doença que pode apresentar um largo espectro de manifestaçôes clínicas, desde formas assintomáticas (sorologia positiva sem manifestaçóes clínicas) à forma clássica, com a presença de hepatoesplenomegalia febril, emagrecimento, pancitopenia e hipergamaglobulinemia, além de importante queda do estado geral. Nas formas assintomáticas, não está indicada a realização de tratamento nem a notificação do caso e, geralmente, tais casos de LV são descobertos em inquéritos sorológicos. Neste estudo, as manifestaçóes clínicas mais frequentes relatadas foram: febre, fraqueza e hepatoesplenomegalia, sintomas estes mais verificados na literatura. ${ }^{5,10,17}$ Fatores ligados ao tempo de diagnóstico podem ocasionar variações na sintomatologia. ${ }^{17}$

Uma das maiores ameaças ao controle da LV em algumas regióes do mundo tem sido a sua interação com o HIV, pois esta tem aparecido como uma importante doença oportunista em áreas endêmicas. A pandemia de HIV/AIDS tem modificado a história natural da LV, aumentando o risco de adoecimento de 100 a 2320 vezes nas áreas endêmicas, reduzindo a eficácia terapêutica e aumentando a possibilidade de recidivas. ${ }^{1}$ Em algumas áreas da Europa meridional, mais de $70 \%$ dos casos de LV em adultos estão associados à infecção pelo HIV., ${ }^{1,2}$

Desde que o primeiro caso de LV associado à infecçáo pelo HIV foi descrito em 1985, a sua frequência vem aumentando rapidamente, principalmente no sul da Europa. Após a introdução da HAART (Hightly Active Antiretroviral Therapy), os casos de coinfecção nos países europeus onde a LV é endêmica têm diminuído; no entanto, a infecção pelo HIV tem se expandido para outros focos maiores de LV. ${ }^{1}$

A coinfecção Leishmania-HIV foi encontrada em 4,6\% dos casos diagnosticados em Aracaju, no período estudado, sendo coerente com o que tem sido relatado na maioria dos países endêmicos (2 e 9\%), mas esta proporção tem aumentado dramaticamente em algumas regióes. De forma geral, considera-se que assim como a incidência mundial da coinfecção é subestimada, no município de Aracaju isso também está ocorrendo, pois boa parte dos pacientes com LV desconhece sua situação sorológica para $\mathrm{HIV}^{1}$. Um fato importante é que da mesma forma que a LV tem passado pelo processo de urbanização, o HIV tem se interiorizado, sendo inevitável o encontro dessas duas endemias.

Com a expansão da LV, torna-se necessário um maior acesso às técnicas de diagnóstico, além da maior sensibilidade dos profissionais na identificação dos seus sinais e sintomas. Neste estudo, 4,5\% dos casos tiveram o seu diagnóstico baseado apenas em evidências clínicas e epidemiológicas, sem a comprovação laboratorial, mas com evolução e resposta terapêutica compatíveis. Outros autores também têm identificado percentual semelhante de casos em que não foi possível a confirmação laboratorial. ${ }^{7,14,18}$

O diagnóstico considerado de certeza ocorre pelo encontro de formas amastigotas do parasito, principalmente pela pesquisa direta em aspirados de medula óssea e/ou mielocultura. Em algumas localidades, esta tem sido a técnica mais empregada, ${ }^{10}$ mas, pela maior praticidade, os métodos imunológicos têm sido cada vez mais utilizados. 
$\mathrm{Na}$ nossa casuística, o exame parasitológico foi realizado em apenas 36,7\% (47) dos casos, com uma positividade de $66,6 \%$. A positividade da microscopia direta em aspirado de medula óssea tem variado em diversos estudos de cerca de 66,0 a $91,8 \%{ }^{10,14,15,18}$

A Reação de Imunofluorescência Indireta (RIFI) foi o exame mais empregado (79,7\%), com a positividade de 90,2\%. Esta tem sido a reação sorológica mais utilizada para o diagnóstico da LV no Brasil, com uma sensibilidade que varia de 55 a 96\%, e especificidade de 70 a 98\%. Sáo consideradas amostras positivas aquelas com titulação a partir de 1:80. ${ }^{3,5,18}$

O Ministério da Saúde (MS) implantou, com o objetivo de agilizar o diagnóstico nas unidades de referência, o teste rk39, um antígeno de leishmanias, que, além de ser um teste não invasivo, tem demonstrado boa sensibilidade (93\%) e especificidade (97\%). ${ }^{19}$ Neste estudo, o teste rK39 foi realizado em 51 casos, com uma positividade de $88,2 \%$, indicando uma grande utilidade para o diagnóstico, principalmente em casos graves, nos quais a demora do diagnóstico pode resultar no óbito do paciente.

Por mais de 70 anos, os antimoniatos pentavalentes foram considerados as drogas de escolha para o tratamento da LV em todo o mundo, mas a emergência de cepas resistentes de leishmanias tem levado à avaliação de outros tratamentos, incluindo o uso das formulaçóes de anfotericina B desoxicolato (convencional) e lipossomal. ${ }^{20}$

Existe, no mundo, uma grande variação na resposta clínica às drogas antileishmanias, assim como uma variação nas recomendações das diferentes regióes afetadas. Os antimoniatos pentavalentes permanecem efetivos e como drogas de escolha na África, na América do Sul e em Bangladesh, Nepal e Índia (exceto Bihar do Norte), numa dose de $20 \mathrm{mg} / \mathrm{kg} /$ dia parenteral, de 21 a 30 dias. $^{21,22}$

O antimoniato pentavalente $\left(\right.$ Glucantime $\left.^{\circledR}\right)$ foi a droga de escolha para o tratamento de $78,15 \%$ (100) dos casos de LV estudados. Apesar de o Glucantime ${ }^{\circledR}$ continuar sendo a droga de primeira escolha para o tratamento da LV no Brasil, o uso das formulaçôes de anfotericina tem sido encorajado em algumas situaçôes, com o intuito de diminuir a letalidade pelo agravo ou pela toxicidade do antimoniato., Além do menor custo, o antimoniato possui a vantagem de poder ser utilizado de forma ambulatorial. ${ }^{3,4,20,21}$

$\mathrm{Na}$ última década, o emprego da anfotericina B lipossomal (Ambisone ${ }^{\circledR}$ ) tem aumentado em todo o mundo como tratamento para LV, pois estudos têm demonstrado melhor eficácia terapêutica, principalmente em áreas onde há resistência ao tratamento com os antimoniatos. Um grande obstáculo para o seu uso ainda é o alto custo. ${ }^{20,21,22}$

Alguns estudos têm consolidado as evidências de que pacientes com LV com mais de 45 anos apresentam um maior risco de morte ou de reaçóes adversas mais severas durante o tratamento com os antimoniatos do que os pacientes mais jovens. ${ }^{23}$

Com o objetivo de diminuir a letalidade por LV foi lançado no Brasil, em 2011, pelo MS, o guia 'Recomendações clínicas para reduçáo da letalidade', no qual se estabelece a indicação das drogas leishmanicidas disponíveis para uso no Brasil. Reforça-se nesse guia a contraindicação do Glucantime ${ }^{\circledR}$ nas gestantes e em pacientes com mais de 50 anos com insuficiência renal, insuficiência hepática ou insuficiência cardíaca. ${ }^{4}$ Verificamos, dessa forma, que apesar da contraindicação, metade dos pacientes com mais de 50 anos teve, neste estudo, a prescriçáo do antimoniato. Em meados de 2013, o MS ampliou a indicação de uso da anfotericina B lipossomal para o tratamento da LV, passando esta a ser considerada a droga de primeira escolha também para menores de um ano de idade; coinfectados com HIV e outras comorbidades ou usando medicamentos que comprometam a imunidade, e falha terapêutica ao antimoniato de N-metil glucamina. A anfotericina B lipossomal para o tratamento da LV é ofertada de forma gratuita pelo Sistema Único de Saúde (SUS) mas, para o seu acesso, o médico solicitante deve preencher uma ficha específica, que pode ser enviada por e-mail (leishmanioses@saude. gov.br) ou digitada diretamente na plataforma do Formsus, acessando-se o endereço eletrônico http://formsus.datasus.gov. $\mathrm{br} /$ site/formulario.php?id_aplicacao $=12043 .{ }^{24}$

No Brasil, a letalidade tem aumentado, variando de 3,4\% (1994) para 6,7\% (2011), apresentando uma letalidade média de 5,8\% nos últimos anos. ${ }^{3,4,25} \mathrm{~A}$ taxa de letalidade no período estudado foi superior $(7,8 \%)$ à média nacional, mas inferior a um estudo anterior em Aracaju, que demonstrou uma letalidade de $8,9 \% .{ }^{13}$

A letalidade variou de acordo com a idade e o sexo, sendo significativamente maior acima dos 40 anos, principalmente na faixa entre 40 e 49 anos, em que foi de 26,7\% ( $p=0,012)$, como observado em alguns estudos. ${ }^{10,23}$ Apesar de uma maior letalidade nas mulheres, a diferença não foi significativa.

Foram identificados como fatores significativamente associados a uma maior letalidade: o edema, as hemorragias e, principalmente, a icterícia. A icterícia à admissão do paciente sugere o envolvimento hepático e tem sido identificada como um fator de gravidade, o qual pode ser piorado com o uso do Glucantime ${ }^{\circledR}$, pela sua hepatoxidade. ${ }^{10,23,25}$ Outros autores 
também têm identificado a presença de edema como fator de risco para óbito, podendo estar associado ao envolvimento renal e à hipoalbuminemia. ${ }^{10,26}$

Reconhece-se como uma limitação deste estudo a utilização de dados secundários das fichas de notificação de LV, tornando-se possível a análise apenas das variáveis nestas preenchidas.

\section{Conclusões}

O município de Aracaju pode ser classificado como área de transmissão intensa da LV, com uma média superior a 4,4 casos nos últimos três anos. ${ }^{3}$ Apesar da prevalência da doença em Aracaju e da sua alta cobertura pela Estratégia Saúde da Família (ESF), verifica-se que a maioria dos casos ainda é diagnosticada na rede hospitalar, podendo indicar um retardo no seu diagnóstico, com possibilidade de aumento da gravidade e consequente alta letalidade.

Para o enfrentamento adequado da LV, além das medidas relacionadas às intervençóes ambientais e ao controle vetorial, é essencial a constante atualização dos profissionais de saúde, principalmente dos médicos da atenção primária (APS) e das unidades de pronto-atendimento, para que consigam identificar precocemente os casos suspeitos, a fim de que diagnóstico e tratamento ocorram de forma oportuna. Estudos têm demonstrado que açôes integradas de programas de treinamento das equipes associadas a ações de controle e integração dos serviços podem colaborar para a redução da letalidade. ${ }^{27}$

É fundamental que o profissional esteja alerta para os riscos e agravos existentes no seu território. Quadros clínicos com febres intermitentes, associados à fraqueza e hepatoesplenomegalia, devem ter a pesquisa de LV como prioridade, respeitando-se a epidemiologia local, mas atentos para as possíveis alterações no padrão de distribuição desta endemia.

\section{Agradecimentos}

Agradecemos à equipe da Diretoria de Vigilância Epidemiológica da Secretaria de Estado da Saúde de Sergipe pela disponibilização dos dados.

\section{Referências}

1. Alvar J, Aparicio P, Aseffa A, Boer MD, Canavete C, Dedet JP, et al. The relationship between leishmaniasis and AIDS: the second 10 years. Clin Microbiol Rev. 2008;21(2):334-359. http://dx.doi.org/10.1128/CMR.00061-07

2. World Health Organization (WHO). Report of the Fifth Consultative Meeting on Leishmania/HIV Coinfection. Addis Ababa: WHO; 2007. Disponível em: http://www.who.int/leishmaniasis/resources/Leishmaniasis_hiv_coinfection5.pdf

3. Ministério da Saúde (BR). Guia de vigilância epidemiológica: Leishmaniose visceral. $7^{a}$ ed. Brasília: Ministério da Saúde; 2009.

4. Ministério da Saúde (BR). Leishmaniose visceral: recomendações clínicas para redução da letalidade. Brasília: Ministério da Saúde; 2011.

5. Albuquerque PLMM, Silva Júnior GB, Freire CCF, Oliveira SBC, Almeida DM, Silva HF, et al. Urbanization of visceral leishmaniasis (kala-azar) in Fortaleza, Ceará, Brazil. Rev Panam Salud Publica. 2009;26(4):330-3. http://dx.doi.org/10.1590/S1020-49892009001000007

6. Marzochi MCA, Fagundes A, Andrade MV, Souza MB, Madeira MF, Mouta-Confort E, et al. Visceral leishmaniasis in Rio de Janeiro, Brazil: eco-epidemiological aspects and control. Rev Soc Bras Med Trop. 2009;42(5):570-580. http://dx.doi.org/10.1590/S0037-86822009000500017

7. Silva AR, Tauil PL, Cavalcante MNS, Medeiros MN, Pires BN, Gonçalves EGR. Situação epidemiológica da leishmaniose visceral na llha de São Luís, Estado do Maranhão. Rev Soc Bras Med Trop. 2008;41(4):358-64. http://dx.doi.org/10.1590/S0037-86822008000400007

8. Maia-Elkhoury ANS, Alves WA, Sousa-Gomes ML, Sena JM, Luna EA. Visceral leishmaniasis in Brazil: trends and challenges. Cad Saúde Pública. 2008;24(12):2941-47. http://dx.doi.org/10.1590/S0102-311X2008001200024

9. Mestre GLC, Fontes CJF. A expansão da epidemia da visceral leishmaniose visceral no Estado de Mato Grosso, 1998-2005. Rev Soc Bras Med Trop. 2007;40(1):42-48. http://dx.doi.org/10.1590/S0037-86822007000100008

10. Oliveira JM, Fernandes AC, Dorval MEC, Alves TP, Fernandes TD, Oshiro ET, et al. Mortalidade por leishmaniose visceral: aspectos clínicos e laboratoriais. Rev Soc Bras Med Trop. 2010;43(2):188-93. http://dx.doi.org/10.1590/S0037-86822010000200016 
11. Tavares LMSA, Tavares ED. Incidência, distribuição geográfica e aspectos ambientais das áreas endêmicas da Leishmaniose Visceral em Sergipe. Inf Epidemiol SUS. 1999;8(1):47-52. http://dx.doi.org/10.5123/S0104-16731999000100006

12. Jeraldo VLS, Góes MAO, Casanova C, Melo CM, Araújo ED, Brandão-Filho SP, et al. Sandfly fauna in an area endemic for visceral leishmaiasis in Aracaju, State of Sergipe, Northeast Brasil. Rev Soc Bras Med Trop. 2012;45(3):318-322. http://dx.doi.org/10.1590/S0037-86822012000300008

13. Góes MAO, Melo CM, Jeraldo VLS. Time series of visceral leishmaniasis in Aracaju, state of Sergipe, Brazil (1999 to 2008): human and canine aspects. Rev Bras Epidemiol. 2012;15(2):298-307. http://dx.doi.org/10.1590/S1415-790X2012000200007

14. Pastorino AC, Jacob CMA, Oselka GW, Carneiro-Sampaio MMS. Leishmaniose visceral: aspectos clínicos e laboratoriais. J Pediatr. 2002;78(2):120-7. http://dx.doi.org/10.1590/S0021-75572002000200010

15. Xavier-Gomes LM, Costa WB, Prado PF, Oliveira-Campos M, Leite MTS. Características e epidemiológicas da leishmaniose visceral em crianças internadas em um hospital universitário de referência no norte de Minas Gerais, Brasil. Rev Bras Epidemiol. 2009;12(4):549-55. http://dx.doi.org/10.1590/S1415-790X2009000400005

16. Gil-Prieto R, Walter S, Alvar J, Miguel AG. Epidemiology of leishmaniasis in Spain based on hospitalization records (1997-2008). Am J Trop Med Hyg. 2011;85(5):820-825. http://dx.doi.org/10.4269/ajtmh.2011.11-0310

17. Nunes WS, Araújo SR, Calheiros CML. Epidemiological profile of leishmaniasis at a reference service in the State of Alagoas, Brazil, from January 2000 to September 2008. Braz J Infect Dis. 2010;14(4):342-5. http://dx.doi.org/10.1590/S1413-86702010000400005

18. Rigo RS, Rigo L, Honer MR. Aspectos Clínicos e Laboratoriais na Leishmaniose Visceral Americana. J Bras Nefrol. 2009;31(1):48-54. Disponível em: http://www.jbn.org.br/detalhe_artigo.asp?id=9

19. Romero GAS, Boelaert M. Control of visceral leishmaniasis in Latin America - A systematic review. PLoS Negl Trop Dis. 2010;4(1):1-17. http://dx.doi.org/10.1371/journal.pntd.0000584

20. Rosenthal E, Delaunay P, Jeandel PY, Haas H, Pomares-Estran C, Marty P. Liposomal amphotericin B as treatment for visceral leishmaniasis in Europe, 2009. Med Mal Infect 2009;39(10):741-4. http://dx.doi.org/10.1016/j.medmal.2009.05.001

21. Bern C, Adler-Moore J, Berenguer J, Boelaert M, Den Boer M, Davidson RN, et al. Liposomal amphotericin B for the treatment of visceral leishmaniasis. Clin Infect Dis. 2006;43(7):917-24. http://dx.doi.org/10.1086/507530

22. Sundar S, Chakravarty J. Liposomal amphotericin B and leishmaniasis: dose and response. J Glob Infect Dis. 2010;2(2):159-166. http://dx.doi.org/10.4103/0974-777X.62886

23. Chappuis F, Alirol E, Worku DT, Mueller Y, Ritmeijer K. High mortality among older patients treated with pentavalent antimonials for visceral leishmaniasis in East Africa and rationale for switch to liposomal amphotericin B. Antimicrob Agents Chemother. 2011;55(1):455-456. http://dx.doi.org/10.1128/AAC.01298-10

24. Ministério da Saúde (BR). SVS divulga novo protocolo de tratamento da leishmaniose visceral. Portal Saúde. 2013 Sep 25 [acesso em 2013 Sept 20]. Disponível em http://portalsaude.saude.gov.br/index.php/profissional-e-gestor/vigilancia/noticias-vigilancia/7700-

25. Ministério da Saúde (BR). Secretaria de Vigilância em Saúde. Letalidade por Leishmaniose Visceral no Brasil, 1990 a 2011. Brasília 2012 [acesso em 2013 Dec 28]. Disponível em http://portal.saude.gov.br/portal/arquivos/pdf/2012_11_letalidade_por_Iv_entre_1990_e_2011.pdf

26. Alvarenga DG, Escalda PMF, Costa ASV, Monreal MTFD. Leishmaniose visceral: estudo retrospectivo de fatores associados à letalidade. Rev Soc Bras Med Trop. 2010;43(2):194-7. http://dx.doi.org/10.1590/S0037-86822010000200017

27. Luz ZMP, Carneiro M, Schall V, Rabello A. The organization of health services and visceral leishmaniasis: an integrated intervention to improve diagnosis and treatment. Cad Saúde Pública. 2009;25(5):1177-1184. http://dx.doi.org/10.1590/S0102-311X2009000500024 